Faculty of Science

Faculty Publications

Some Janowski Type Harmonic $q$-Starlike Functions Associated with Symmetrical Points

Muhammad Arif, Omar Barkub, H. M. Srivastava, Saleem Abdullah, \& Sher Afzal Khan

2020

(C) 2020 Arif et al. This article is an open access article distributed under the terms and conditions of the Creative Commons Attribution (CC BY) license.

http://creativecommons.org/licenses/by/4.0/

This article was originally published at:

https://doi.org/10.3390/math8040629

Citation for this paper:

Arif, M., Barkub, O., Srivastava, H. M., Abdullah, S., \& Khan, S. A. (2020). Some Janowski type harmonic $q$-starlike functions associated with symmetrical points. Mathematics, 8(4). https://doi.org/10.3390/math8040629 
Article

\title{
Some Janowski Type Harmonic $q$-Starlike Functions Associated with Symmetrical Points
}

\author{
Muhammad Arif ${ }^{1, *(\mathbb{C})}$, Omar Barkub ${ }^{2}$, Hari Mohan Srivastava ${ }^{3,4,5}\left(\mathbb{D}\right.$, Saleem Abdullah ${ }^{1}(\mathbb{D}$ and \\ Sher Afzal Khan ${ }^{1}$ \\ 1 Faculty of Physical and Numerical Sciences, Abdul Wali Khan University Mardan, Mardan 23200, Pakistan; \\ saleemabdullah@awkum.edu.pk (S.A.); sher.afzal@awkum.edu.pk (S.A.K.) \\ 2 Faculty of Computing and Information Technology, King Abdulaziz University, Rabigh 21911, Jeddah, \\ Saudi Arabia; obarukab@kau.edu.sa \\ 3 Department of Mathematics and Statistics, University of Victoria, Victoria, BC V8W 3R4, Canada; \\ harimsri@uvic.ca \\ 4 Department of Medical Research, China Medical University Hospital, China Medical University, \\ Taichung 40402, Taiwan \\ 5 Department of Mathematics and Informatics, Azerbaijan University, 71 Jeyhun Hajibeyli Street, \\ AZ1007 Baku, Azerbaijan \\ * Correspondence: marifmaths@awkum.edu.pk
}

Received: 11 February 2020; Accepted: 16 April 2020; Published: 19 April 2020

\begin{abstract}
The motive behind this article is to apply the notions of $q$-derivative by introducing some new families of harmonic functions associated with the symmetric circular region. We develop a new criterion for sense preserving and hence the univalency in terms of $q$-differential operator. The necessary and sufficient conditions are established for univalency for this newly defined class. We also discuss some other interesting properties such as distortion limits, convolution preserving, and convexity conditions. Further, by using sufficient inequality, we establish sharp bounds of the real parts of the ratios of harmonic functions to its sequences of partial sums. Some known consequences of the main results are also obtained by varying the parameters.
\end{abstract}

Keywords: harmonic function; Janowski starlike functions; $q$-derivative operator

\section{Introduction}

To better understand the concepts used in our key findings, some of the basic relevant Geometric Function Theory literature needs to be included and studied here. To do this, we begin with notation $\mathcal{H}$ which indicates the harmonic functions class in $\mathfrak{D}:=\mathfrak{D}(1)$, where $\mathfrak{D}(r)=\{z \in \mathbb{C}:|z|<r\}$. Also, let $\mathcal{H}_{0}$ be denoted by the family of functions $f=\mathfrak{h}+\overline{\mathfrak{g}} \in \mathcal{H}$ which have the series expansion:

$$
f(z)=z+\sum_{n=2}^{\infty}\left(a_{n} z^{n}+\overline{b_{n} z^{n}}\right) \quad(z \in \mathfrak{D}),
$$

where $\mathfrak{h}$ and $\mathfrak{g}$ are holomorphic functions with the following series expansion:

$$
\mathfrak{h}(z)=z+\sum_{n=2}^{\infty} a_{n} z^{n}, \mathfrak{g}(z)=\sum_{n=2}^{\infty} b_{n} z^{n}(z \in \mathfrak{D}) .
$$

The series defined in (1) and (2) are convergent in the open unit disc $\mathfrak{D}$. Also, let's select the symbol $\mathcal{S}$ which contains all functions (say $f \in \mathcal{S}$ ) that are univalent holomorphic in $\mathfrak{D}$ and satisfy the relationship $f(0)=f^{\prime}(0)-1=0$. Further, the notation $\mathcal{S}_{\mathcal{H}}$ denotes the family of complex-valued 
functions $f \in \mathcal{H}_{0}$ which are sense preserving and univalent in $\mathfrak{D}$. Clearly, $\mathcal{S}_{\mathcal{H}}$ coincides with the set $\mathcal{S}$ of normalized univalent holomorphic functions if $\mathfrak{g}(z)$ becomes zero in $\mathfrak{D}$. Clunie and Sheil-Small [1] and Sheil-Small [2] studied the class $\mathcal{S}_{\mathcal{H}}$ along with some of their subfamilies. Particularly, they explored and studied the families of starlike harmonic and convex harmonic functions in $\mathfrak{D}$, which are given as follows:

$$
S_{\mathcal{H}}^{*}=\left\{f \in S_{\mathcal{H}}: \frac{\mathcal{D}_{\mathcal{H}} f(z)}{f(z)} \prec \frac{1+z}{1-z}(z \in \mathfrak{D})\right\}
$$

and

$$
S_{\mathcal{H}}^{c}=\left\{f \in S_{\mathcal{H}}: \mathcal{D}_{\mathcal{H}} f(z) \in S_{\mathcal{H}}^{*}(z \in \mathfrak{D})\right\},
$$

where

$$
\mathcal{D}_{\mathcal{H}} f(z)=z \mathfrak{h}^{\prime}(z)-\overline{z \mathfrak{g}^{\prime}(z)} .
$$

Here the symbol " $\prec$ " represents the familiar subordination between holomorphic functions represented by " $f_{1} \prec f_{2}$ " and is defined as; two holomorphic functions $f_{1}: \mathfrak{D} \rightarrow \mathbb{C}$ and $f_{2}: \mathfrak{D} \rightarrow \mathbb{C}$ are connected by the subordination relationship, if a holomorphic function $u$ occurs with the limitations $u(0)=0$ and $|u(z)|<1$, in such a way that $f_{1}(z)=f_{2}(u(z))$. Additionally, if $f_{2}$ is univalent, then we obtain:

$$
f_{1}(z) \prec f_{2}(z) \quad(z \in \mathfrak{D}) \quad \Longleftrightarrow \quad\left[f_{1}(0)=f_{2}(0) \quad \& \quad f_{1}(\mathfrak{D}) \subset f_{2}(\mathfrak{D})\right] .
$$

In 2015, Dziok [3] introduced a family $\mathcal{S}_{\mathcal{H}}^{*}(L, M),(L, M \in \mathbb{C}$ with $L \neq M)$, of Janowski harmonic functions with the help of the concepts established in [4] and this class is defined by;

$$
\mathcal{S}_{\mathcal{H}}^{*}(L, M)=\left\{f \in S_{\mathcal{H}}: \frac{\mathcal{D}_{\mathcal{H}} f(z)}{f(z)} \prec \frac{1+L z}{1+M z}(z \in \mathfrak{D})\right\},
$$

where $\mathcal{D}_{\mathcal{H}} f(z)$ is given by $(3)$. The families $\mathcal{S}_{\mathcal{H}}^{*}(\beta):=\mathcal{S}_{\mathcal{H}}^{*}(1-2 \beta,-1)$ and $\mathcal{S}_{\mathcal{H}}^{c}(\beta):=\mathcal{S}_{\mathcal{H}}^{c}(1-2 \beta,-1)$ with the restriction $0 \leq \beta<1$, were examined by Jahangiri [5] and Ahuja [6]. Moreover, for $\beta=0$, we have the most basic families $\mathcal{S}_{\mathcal{H}}^{*}:=\mathcal{S}_{\mathcal{H}}^{*}(1,-1)$ and $\mathcal{S}_{\mathcal{H}}^{c}:=\mathcal{S}_{\mathcal{H}}^{c}(1,-1)$ of functions $f \in S_{\mathcal{H}}$ which are starlike and convex in $\mathfrak{D}(r)$ respectively, for any $r \in[0,1)$. For more work on harmonic mappings, see [7-10].

Quantum calculus or just $q$-calculus is the classical calculus without the limit notion. This theory was originated by Jackson [11,12]. The researchers were inspired by the learning of $q$-calculus because of its contemporary use in various claims; for example in differential equations, quantum theory, the theory of special functions, analytic number theory, combinatorics, operator theory, numerical analysis, and other related theories, see $[13,14]$. Just as $q$-calculus has been used in other branches of Mathematics, the applications of this concept have been widely used to analyze the geometric nature of various analytical functions in Geometric functions theory. In this regard, Ismail et al. [15] published the first paper by exploring the geometry of $q$-starlike functions. This concept was further expanded by Agrawal and Sahoo [16] by introducing the $q$-starlike functions family with some order. Due to this advancement in functions theory, many researchers were inspired. They made significant contributions which gradually enhanced the attractiveness of this research area for potential researchers. For more literature on quantum calculus, see [17-25].

For the given $q \in] 0,1[$, the $q$-analog derivative of $f$ is defined as:

$$
\mathcal{D}_{q} f(z)=\frac{f(z)-f(q z)}{z(1-q)},(z \in \mathfrak{D}) .
$$

Making use of (1) and (4), one can get easily for $n \in \mathbb{N}$

$$
\begin{aligned}
\mathcal{D}_{q} f(z) & =\mathcal{D}_{q} \mathfrak{h}(z)+\overline{\mathcal{D}_{q} \mathfrak{g}(z)} \\
& =1+\sum_{n=2}^{\infty}[n]_{q} a_{n} z^{n-1}+\sum_{n=2}^{\infty}[n]_{q} \overline{b_{n} z^{n-1}},
\end{aligned}
$$


where

$$
[n]_{q}=\frac{1-q^{n}}{1-q}=1+\sum_{k=1}^{n-1} q^{k},[0]_{q}=0
$$

In 1956 Sakaguchi [26] established the family $\mathcal{S}_{s}^{*}$ of holomorphic univalent functions in $\mathfrak{D}$ which are starlike with respect to symmetrical points; a holomorphic function $f$ is said to be starlike with respect to symmetric points if

$$
\frac{z f^{\prime}(z)}{f(z)-f(-z)} \prec \frac{1+z}{1-z}, \quad(z \in \mathfrak{D}) .
$$

Using this idea of Sakaguchi, Cho and Dziok [27] recently introduced the family $\mathcal{H} \mathcal{S}_{s}^{*}(L, M)$ of functions $f \in \mathcal{S}_{\mathcal{H}}$ such that

$$
\frac{2 \mathcal{D}_{\mathcal{H}} f(z)}{f(z)-f(-z)} \prec \frac{1+L z}{1+M z}, \quad(z \in \mathfrak{D}),
$$

where $\mathcal{D}_{\mathcal{H}} f(z)$ is given by (3). They investigated some useful properties such as coefficient estimates, subordination properties, distortion theorems, and integral representation for the functions belongs to the family $\mathcal{H S}_{s}^{*}(L, M)$. The family $\mathcal{H} \mathcal{S}_{s}^{*}(L, M)$ generalizes various known families discussed earlier by many researchers, see [28-31]. Motivated by the above work, we now define the following subfamilies of Janowski harmonic mappings involving $q$-derivative. Before definition and to prevent repetitions we will assume (except as otherwise stated) that

$$
-1 \leq M<L \leq 1, q \in] 0,1[, 0 \leq \beta<1 \text { and }-1 \leq y<x \leq 1 .
$$

Definition 1. Let $\mathcal{H} \mathcal{S}_{s}^{*}(x, y, q, L, M)$ be the family of functions $f \in \mathcal{S}_{\mathcal{H}}$ such that

$$
\frac{(x-y) \mathcal{D}_{\mathcal{H}}^{q} f(z)}{f(x z)-f(y z)} \prec \frac{1+L z}{1+M z},
$$

where

$$
\mathcal{D}_{\mathcal{H}}^{q} f(z)=z D_{q} \mathfrak{h}(z)-\overline{z D_{q} \mathfrak{g}(z)} \quad(z \in \mathfrak{D}) .
$$

Additionally let's describe

$$
\mathcal{H S}_{S}^{c}(x, y, q, L, M):=\left\{f \in \mathcal{S}_{\mathcal{H}}: \mathcal{D}_{\mathcal{H}}^{q} f(z) \in \mathcal{H S}_{S}^{*}(x, y, q, L, M)\right\} .
$$

\section{Special Cases:}

(i). The below two families

$$
\begin{aligned}
& \mathcal{H S}_{s}^{*}(L, M):=\lim _{q \rightarrow 1^{-}} \mathcal{H} \mathcal{S}_{s}^{*}(1,-1, q, L, M), \\
& \mathcal{H S}_{S}^{c}(L, M):=\lim _{q \rightarrow 1^{-}} \mathcal{H} \mathcal{S}_{S}^{c}(1,-1, q, L, M),
\end{aligned}
$$

were investigated by Cho and Dziok [27] recently and further by setting $L=1-2 \beta, M=-1$, we achieve

$$
\mathcal{H S}_{s}^{*}(\beta):=\mathcal{H S}_{s}^{*}(1-2 \beta,-1),
$$

which was studied by Ahuja and Jahangiri [28] (see also [29,30]) .

(ii). For $b \in \mathbb{C} \backslash\{0\}$, the family

$$
\mathcal{H} \mathcal{S}_{\mathcal{S}}^{*}(b, \beta):=\lim _{q \rightarrow 1^{-}} \mathcal{H} \mathcal{S}_{S}^{*}(1,-1, q, 2 b(\beta-1)+1,1),
$$


was examined by Janteng and Halim [31].

(iii). Also the following families

$$
\begin{aligned}
& \mathcal{S}_{\mathcal{H}}^{*}(L, M):=\lim _{q \rightarrow 1^{-}} \mathcal{H} \mathcal{S}_{s}^{*}(1,0, q, L, M), \\
& \mathcal{S}_{\mathcal{H}}^{c}(L, M):=\lim _{q \rightarrow 1^{-}} \mathcal{H} \mathcal{S}_{\mathcal{S}}^{c}(1,0, q, L, M),
\end{aligned}
$$

were discussed by Dziok in [3]. Similarly, the families $\mathcal{S}_{\mathcal{H}}^{*}(\beta):=\mathcal{S}_{\mathcal{H}}^{*}(1-2 \beta,-1)$ and $\mathcal{S}_{\mathcal{H}}^{c}(\beta):=$ $\mathcal{S}_{\mathcal{H}}^{c}(1-2 \beta,-1)$ were explored in $[5,6]$.

In this article, we obtain some interesting properties for the newly described classes including necessary and sufficient conditions, distortion limits, problems with partial sums, convolutions and convexity conditions. Several implications of the key results are also given.

\section{Necessary and Sufficient Conditions}

Theorem 1. Let $f \in \mathcal{H}$ be expressed in series expansion (1) and if the following inequality

$$
\sum_{n=2}^{\infty}\left(\sigma_{n}\left|a_{n}\right|+v_{n}\left|b_{n}\right|\right) \leq L-M,
$$

holds, then $f \in \mathcal{H S}_{s}^{*}(x, y, q, L, M)$ with

$$
\begin{aligned}
& \sigma_{n}=\left|[n]_{q}-\frac{x^{n}-y^{n}}{x-y}\right|+\left|M[n]_{q}-L \frac{x^{n}-y^{n}}{x-y}\right|, \\
& v_{n}=\left|[n]_{q}+\frac{x^{n}-y^{n}}{x-y}\right|+\left|M[n]_{q}+L \frac{x^{n}-y^{n}}{x-y}\right| .
\end{aligned}
$$

Proof. For $f(z)=z$, we have $h(z)=z$ and $g(z)=0$. To prove that $f$ is sense preserving and locally univalent, we consider

$$
\left|h^{\prime}(z)\right|-\left|g^{\prime}(z)\right|=1>0 .
$$

Hence, due to the result of Lewy [9], the function $f$ in $\mathfrak{D}$ is thus orientation-preserving and locally univalent. Now we show that $f$ is univalent in $\mathfrak{D}$. Let $z_{1}, z_{2} \in \mathfrak{D}$ and $z_{1} \neq z_{2}$. Then

$$
\left|f\left(z_{1}\right)-f\left(z_{2}\right)\right|=\left|z_{1}-z_{2}\right| \geq 0 .
$$

To prove that $f \in \mathcal{H} \mathcal{S}_{s}^{*}(x, y, q, L, M)$, we have to show that

$$
\left|\frac{\frac{(x-y) \mathcal{D}_{\mathcal{H}}^{q} f(z)}{f(x z)-f(x z)}-1}{M \frac{(x-y) \mathcal{D}_{\mathcal{H}}^{q} f(z)}{f(x z)-f(x z)}-L}\right|<1 .
$$

It is easy to see that $\mathcal{D}_{\mathcal{H}}^{q} f(z)=z$ and $L-M>0$. This implies that

$$
\left|\frac{\frac{(x-y) \mathcal{D}_{\mathcal{H}}^{q} f(z)}{f(x z)-f(x z)}-1}{M \frac{(x-y) \mathcal{D}_{\mathcal{H}}^{q} f(z)}{f(x z)-f(x z)}-L}\right|=\left|\frac{1-1}{M-L}\right|=0<1 .
$$

Hence, $f \in \mathcal{H S}_{S}^{*}(x, y, q, L, M)$. Now let $f \in \mathcal{H}$ has the expansion form (1) and then let we presume that there is $n \geq 2$ so that $a_{n} \neq 0$ or $b_{n} \neq 0$. Also by using

$$
\frac{x^{n}-y^{n}}{x-y}=x^{n-1}+x^{n-2} y+x^{n-3} y^{2}+\cdots+y^{n-1} \leq n,
$$


and

$$
[n]_{q}=\left(1+\sum_{k=1}^{n-1} q^{k}\right)>1
$$

we have

$$
\begin{aligned}
\frac{\sigma_{n}}{L-M} & =\frac{1}{L-M}\left\{\left|[n]_{q}-\frac{x^{n}-y^{n}}{x-y}\right|+\left|M[n]_{q}-L \frac{x^{n}-y^{n}}{x-y}\right|\right\} \\
& \geq \frac{|1-n|+|M-L n|}{L-M}=\frac{(1+L) n-(M+1)}{L-M} \\
& \geq \frac{(1+L) n-(M+1) n}{L-M}=n, \text { for all } n \geq 2 .
\end{aligned}
$$

Similarly $\frac{v_{n}}{L-M} \geq n$ for $n \geq 2$. Therefore by using (8) along with the above facts, we obtain

$$
\sum_{n=2}^{\infty}\left(n\left|a_{n}\right|+n\left|b_{n}\right|\right) \leq 1
$$

and so

$$
\begin{aligned}
\left|\mathfrak{h}^{\prime}(z)\right|-\left|\mathfrak{g}^{\prime}(z)\right| & \geq 1-\sum_{n=2}^{\infty} n\left|a_{n}\right|\left|z^{n}\right|-\sum_{n=2}^{\infty} n\left|b_{n}\right|\left|z^{n}\right| \\
& \geq 1-|z|\left(\sum_{n=2}^{\infty} n\left|a_{n}\right|+\sum_{n=2}^{\infty} n\left|b_{n}\right|\right) \\
& \geq 1-\frac{|z|}{L-M}\left(\sum_{n=2}^{\infty} \sigma_{n}\left|a_{n}\right|+\sum_{n=2}^{\infty} v_{n}\left|b_{n}\right|\right) \\
& \geq 1-|z|>0,(z \in \mathfrak{D}) .
\end{aligned}
$$

Now by the result proved by Lewy [9], the function $f$ in $\mathfrak{D}$ is thus orientation-preserving and locally univalent. Further, if $z_{1}, z_{2} \in \mathfrak{D}$ with $z_{1} \neq z_{2}$, then

$$
\left|\frac{z_{1}^{n}-z_{2}^{n}}{z_{1}-z_{2}}\right|=\sum_{k=1}^{n}\left|z_{1}\right|^{k-1}\left|z_{2}\right|^{n-k} \leq n, \text { for } n \geq 2
$$

Hence, by the virtue of (11) and (12) along with the triangle inequality, we have

$$
\begin{aligned}
\left|f\left(z_{1}\right)-f\left(z_{2}\right)\right| & \geq\left|\mathfrak{h}\left(z_{1}\right)-\mathfrak{h}\left(z_{2}\right)\right|-\left|\mathfrak{g}\left(z_{1}\right)-\mathfrak{g}\left(z_{2}\right)\right| \\
& \geq\left|\left(z_{1}-z_{2}\right)+\sum_{n=2}^{\infty}\left(z_{1}^{n}-z_{2}^{n}\right) a_{n}\right|-\left|\sum_{n=2}^{\infty} \overline{\left(z_{1}^{n}-z_{2}^{n}\right) b_{n}}\right| \\
& \geq\left|z_{1}-z_{2}\right|-\sum_{n=2}^{\infty}\left|a_{n}\left(z_{1}^{n}-z_{2}^{n}\right)\right|-\sum_{n=2}^{\infty}\left|\overline{b_{n}}\right|\left|\overline{z_{1}^{n}-z_{2}^{n}}\right| \\
& =\left|z_{1}-z_{2}\right|-\sum_{n=2}^{\infty}\left|a_{n}\right|\left|\left(z_{1}^{n}-z_{2}^{n}\right)\right|-\sum_{n=2}^{\infty}\left|b_{n}\right|\left|z_{1}^{n}-z_{2}^{n}\right| \\
& =\left|\left(z_{1}-z_{2}\right)\right|\left(1-\sum_{n=2}^{\infty}\left|\frac{z_{1}^{n}-z_{2}^{n}}{z_{1}-z_{2}}\right|\left|a_{n}\right|-\sum_{n=2}^{\infty}\left|\frac{z_{1}^{n}-z_{2}^{n}}{z_{1}-z_{2}}\right|\left|b_{n}\right|\right) \\
& \geq\left|\left(z_{1}-z_{2}\right)\right|\left(1-\sum_{n=2}^{\infty} n\left|a_{n}\right|-\sum_{n=2}^{\infty} n\left|b_{n}\right|\right) \geq 0 .
\end{aligned}
$$

This yields that $f$ is univalent in $\mathfrak{D}$ and so $f \in \mathcal{S}_{\mathcal{H}}$. Thus $f \in \mathcal{H S}_{s}^{*}(x, y, q, L, M)$ if and only if a holomorphic function $u$ occurs with $u(0)=0$ and $|u(z)|<1(z \in \mathfrak{D})$ so that 


$$
\frac{(x-y) \mathcal{D}_{\mathcal{H}}^{q} f(z)}{f(x z)-f(y z)}=\frac{1+L u(z)}{1+M u(z)} \quad(z \in \mathfrak{D})
$$

or, alternatively

$$
\left|\frac{\frac{(x-y) \mathcal{D}_{\mathcal{H}}^{q} f(z)}{f(x z)-f(y z)}-1}{M \frac{(x-y) \mathcal{D}_{\mathcal{H}}^{q} f(z)}{f(x z)-f(y z)}-L}\right|<1 \quad(z \in \mathfrak{D}) .
$$

Hence, it is enough to establish that

$$
\left|\mathcal{D}_{\mathcal{H}}^{q} f(z)-\frac{(f(x z)-f(y z))}{(x-y)}\right|-\left|M \mathcal{D}_{\mathcal{H}}^{q} f(z)-L \frac{(f(x z)-f(y z))}{(x-y)}\right|<0,
$$

for $z \in \mathfrak{D} \backslash\{0\}$. Setting $|z|=r(0<r<1)$, we have

$$
\begin{aligned}
& \left|\mathcal{D}_{\mathcal{H}}^{q} f(z)-\frac{(f(x z)-f(y z))}{(x-y)}\right|-\left|M \mathcal{D}_{\mathcal{H}}^{q} f(z)-L \frac{(f(x z)-f(y z))}{(x-y)}\right|= \\
& \left|\sum_{n=2}^{\infty}\left([n]_{q}-\frac{x^{n}-y^{n}}{x-y}\right) a_{n} z^{n}-\left([n]_{q}+\frac{x^{n}-y^{n}}{x-y}\right) \frac{b_{n} z^{n}}{}\right|- \\
& -\left|(M-L) z+\sum_{n=2}^{\infty}\left(M[n]_{q}-L \frac{x^{n}-y^{n}}{x-y}\right) a_{n} z^{n}-\left(M[n]_{q}+L \frac{x^{n}-y^{n}}{x-y}\right) \overline{b_{n} z^{n}}\right| \\
\leq & \sum_{n=2}^{\infty}\left|[n]_{q}-\frac{x^{n}-y^{n}}{x-y}\right|\left|a_{n}\right| r^{n}+\left|[n]_{q}+\frac{x^{n}-y^{n}}{x-y}\right|\left|b_{n}\right| r^{n}-(L-M) r+ \\
& +\sum_{n=2}^{\infty}\left|M[n]_{q}-L \frac{x^{n}-y^{n}}{x-y}\right|\left|a_{n}\right| r^{n}+\left|M[n]_{q}+L \frac{x^{n}-y^{n}}{x-y}\right|\left|b_{n}\right| r^{n} \\
\leq & \left\{\sum_{n=2}^{\infty}\left(\sigma_{n}\left|a_{n}\right|+v_{n}\left|b_{n}\right|\right) r^{n-1}-(L-M)\right\} r<0,
\end{aligned}
$$

where we have used (8). Thus $f \in \mathcal{S}_{\mathcal{H}}^{*}(x, y, q, L, M)$.

Substituting particular values of the parameters used in this result, we achieve the following corollaries:

Corollary 1. Let $f \in \mathcal{H}$ be expressed in the series expansion (1) and if the inequality

$$
\sum_{n=2}^{\infty}\left(\sigma_{n}\left|a_{n}\right|+v_{n}\left|b_{n}\right|\right) \leq L-M
$$

is satisfied with

$$
\begin{aligned}
& \sigma_{n}=\left|n-\frac{1-(-1)^{n}}{2}\right|+\left|M n-L \frac{1-(-1)^{n}}{2}\right|, \\
& v_{n}=\left|n+\frac{1-(-1)^{n}}{2}\right|+\left|M n+L \frac{1-(-1)^{n}}{2}\right|
\end{aligned}
$$

then $f \in \mathcal{H S}_{\mathcal{S}}^{*}(L, M)$.

Proof. The result follows by taking $s=1, t=-1$ and $q \rightarrow 1^{-}$in the above Theorem.

Corollary 2. Let $f \in \mathcal{H}$ be expressed in the series expansion (1) and if

$$
\sum_{n=2}^{\infty}\left(\sigma_{n}\left|a_{n}\right|+v_{n}\left|b_{n}\right|\right) \leq L-M
$$


holds true with

$$
\begin{aligned}
& \sigma_{n}=|n-1|+|M n-L|, \\
& v_{n}=|n+1|+|M n+L|,
\end{aligned}
$$

then $f \in \mathcal{S}_{\mathcal{H}}^{*}(L, M)$.

Proof. By putting $s=1, t=0$ and $q \rightarrow 1^{-}$in the above Theorem, we obtain the required result.

Motivated by the work of Silverman [32], we now establish the family $\tau^{\lambda}, \lambda \in\{0,1\}$ of functions $f \in \mathcal{H}_{0}$ of the form (1) such that

$$
a_{n}=-\left|a_{n}\right|, \quad b_{n}=(-1)^{\lambda}\left|b_{n}\right|, \forall n=2,3, \cdots .
$$

Thus (1) and (2) yields $f(z)=\mathfrak{h}(z)+\overline{\mathfrak{g}}(z)$ with

$$
\mathfrak{h}(z)=z-\sum_{n=2}^{\infty}\left|a_{n}\right| z^{n}, \overline{\mathfrak{g}}(z)=(-1)^{\lambda} \sum_{n=2}^{\infty}\left|b_{n}\right| \overline{z^{n}}(z \in \mathfrak{D}) .
$$

Also, we define the families

$$
\begin{aligned}
& \mathcal{S}_{\tau}^{*}(x, y, q, L, M)=\tau^{0} \cap \mathcal{S}_{\mathcal{H}}^{*}(x, y, q, L, M), \\
& \mathcal{S}_{\tau}^{c}(x, y, q, L, M)=\tau^{1} \cap \mathcal{S}_{\mathcal{H}}^{c}(x, y, q, L, M) .
\end{aligned}
$$

Now we are going to confirm that the condition (8) is also sufficient for $f \in \mathcal{S}_{\tau}^{*}(x, y, q, L, M)$.

Theorem 2. Let $f \in \tau^{0}$ has the power series expansion (14). Then $f \in \mathcal{S}_{\tau}^{*}(x, y, q, L, M)$ if and only if (8) holds true.

Proof. To prove our result, it is enough to establish that each function $f \in \mathcal{S}_{\tau}^{*}(x, y, q, L, M)$ satisfies the relation (8). Let $f \in \mathcal{S}_{\tau}^{*}(x, y, q, L, M)$. Then it must satisfy (13). That is; for $z \in \mathfrak{D}$

$$
\left|\frac{-\sum_{n=2}^{\infty}\left([n]_{q}-\frac{x^{n}-y^{n}}{x-y}\right)\left|a_{n}\right| z^{n}-\left([n]_{q}+\frac{x^{n}-y^{n}}{(x-y)}\right)\left|b_{n}\right| \overline{z^{n}}}{(M-L) z-\sum_{n=2}^{\infty}\left(M[n]_{q}-L \frac{x^{n}-y^{n}}{x-y}\right)\left|a_{n}\right| z^{n}-\left(M[n]_{q}+L \frac{x^{n}-y^{n}}{x-y}\right)\left|b_{n}\right| \bar{z}^{n}}\right|<1 .
$$

Setting $z=r(r \in(0,1))$, we have

$$
\frac{\sum_{n=2}^{\infty}\left(\left|[n]_{q}-\frac{x^{n}-y^{n}}{x-y}\right|\right)\left|a_{n}\right| r^{n-1}+\left(\left|[n]_{q}+\frac{x^{n}-y^{n}}{x-y}\right|\right)\left|b_{n}\right| r^{n-1}}{(L-M)-\left\{\sum_{n=2}^{\infty}\left(\left|M[n]_{q}-L \frac{x^{n}-y^{n}}{x-y}\right|\right)\left|a_{n}\right| r^{n-1}-\left(\left|M[n]_{q}+L \frac{x^{n}-y^{n}}{x-y}\right|\right)\left|b_{n}\right| r^{n-1}\right\}}<1 .
$$

It is clear that for $r \in(0,1)$, the denominator of left hand side of (15) can not be zero. Further, it is positive for $r=0$. Thus from (15), we get

$$
\sum_{n=2}^{\infty}\left(\sigma_{n}\left|a_{n}\right|+v_{n}\left|b_{n}\right|\right) r^{n-1} \leq(L-M), \quad(0 \leq r<1) .
$$

It is also clear that the partial sums sequence $\left\{S_{n}\right\}$ connected with the series $\sum_{n=2}^{\infty}\left(\sigma_{n}\left|a_{n}\right|+v_{n}\left|b_{n}\right|\right)$ is a non-decreasing sequence and with the use of $(16)$, it is bounded by $(L-M)$. So $\left\{S_{n}\right\}$ is convergent and

$$
\sum_{n=2}^{\infty}\left(\sigma_{n}\left|a_{n}\right|+v_{n}\left|b_{n}\right|\right) r^{n-1}=\lim _{n \rightarrow \infty} S_{n} \leq(L-M),
$$


which yields assertion (8).

Example 1. Consider the function

$$
G(z)=z-\sum_{n=2}^{\infty}\left(\frac{L-M}{2^{n} \sigma_{n}} z^{n}+\frac{L-M}{2^{n} v_{n}} \bar{z}^{n}\right),(z \in \mathfrak{D}),
$$

then we can easily obtain

$$
\begin{aligned}
\sum_{n=2}^{\infty}\left(\sigma_{n}\left|a_{n}\right|+v_{n}\left|b_{n}\right|\right) & =\sum_{n=2}^{\infty}\left(\frac{L-M}{2^{n}}+\frac{L-M}{2^{n}}\right) \\
& =(L-M) \sum_{n=2}^{\infty} \frac{1}{2^{n-1}}=(L-M) .
\end{aligned}
$$

Hence, $G \in \mathcal{S}_{\tau}^{*}(x, y, q, L, M)$.

By using the above facts, the following two results are easily obtained.

Corollary 3. Let $f \in \mathcal{H}_{0}$ be expressed in series expansion form (1) and if

$$
\sum_{n=2}^{\infty}[n]_{q}\left(\sigma_{n}\left|a_{n}\right|+v_{n}\left|b_{n}\right|\right) \leq(L-M)
$$

then $f \in \mathcal{S}_{\mathcal{H}}^{c}(x, y, q, L, M)$.

Proof. Let $f \in \mathcal{H}_{0}$ be of the form (1) and set

$$
F(z)=\mathcal{D}_{\mathcal{H}}^{q} f(z)=z+\sum_{n=2}^{\infty}\left(a_{n}^{\prime} z^{n}+\overline{b_{n}^{\prime} z^{n}}\right)
$$

with

$$
a_{n}^{\prime}=[n]_{q} a_{n}, b_{n}^{\prime}=-[n]_{q} b_{n} .
$$

Then by using inequality (17) together with Theorem 1 , we obtain $F(z) \in \mathcal{H S}_{s}^{*}(x, y, q, L, M)$ and hence by Alexandar type relation

$$
f(z) \in \mathcal{H S}_{\mathcal{S}}^{c}(x, y, q, L, M) \Leftrightarrow \mathcal{D}_{\mathcal{H}}^{q} f(z) \in \mathcal{H} \mathcal{S}_{s}^{*}(x, y, q, L, M),
$$

we get the required result.

Corollary 4. Let $f \in \tau^{1}$ be the series expansion form (14). Then $f \in \mathcal{S}_{\tau}^{c}(x, y, q, L, M)$ if and only if (17) is satisfied.

Proof. Using the relation (18) and Theorem 2, we easily get the required result.

\section{Investigation of Partial Sums Problems}

In this section, we study the partial sums of some harmonic functions that belong to the family $\mathcal{S}_{\mathcal{H}}^{*}(x, y, q, L, M)$. We develop certain new results which provide relationship between harmonic functions to its sequences of partial sums.

Let $f=\mathfrak{h}+\overline{\mathfrak{g}}$ with $\mathfrak{h}$ and $\mathfrak{g}$ are expressed in the form (2). Then the sequences of partial sum of functions $f$ are given by 


$$
\begin{aligned}
& S_{t}(f)=z+\sum_{n=2}^{t} a_{n} z^{n}+\sum_{n=2}^{\infty} b_{n} \bar{z}^{n}:=S_{t}(\mathfrak{h})+\overline{\mathfrak{g}} \\
& S_{l}(f)=z+\sum_{n=2}^{\infty} a_{n} z^{n}+\sum_{n=2}^{l} b_{n} \bar{z}^{n}:=S_{l}(\overline{\mathfrak{g}})+\mathfrak{h},
\end{aligned}
$$

and

$$
S_{t, l}(f)=z+\sum_{n=2}^{t} a_{n} z^{n}+\sum_{n=2}^{l} b_{n} \bar{z}^{n}:=S_{t}(\mathfrak{h})+S_{l}(\overline{\mathfrak{g}}) .
$$

Here we determine sharp lower bounds of

$$
\operatorname{Re}\left(\frac{f(z)}{S_{t}(f)}\right) \& \operatorname{Re}\left(\frac{S_{t}(f)}{f(z)}\right), \operatorname{Re}\left(\frac{f(z)}{S_{l}(f)}\right) \& \operatorname{Re}\left(\frac{S_{l}(f)}{f(z)}\right),
$$

and

$$
\operatorname{Re}\left(\frac{f(z)}{S_{t, l}(f)}\right) \& \operatorname{Re}\left(\frac{S_{t, l}(f)}{f(z)}\right)
$$

Theorem 3. Let $f$ be of the form (1). If $f$ satisfies the condition (8), then

$$
\operatorname{Re}\left(\frac{f(z)}{S_{t}(f)}\right) \geq \frac{d_{t+1}-L+M}{d_{t+1}}
$$

and

$$
\operatorname{Re}\left(\frac{S_{t}(f)}{f(z)}\right) \geq \frac{d_{t+1}}{d_{t+1}+L-M^{\prime}}
$$

where

$$
d_{n}=\min \left(\sigma_{n}, v_{n}\right),
$$

and

$$
d_{n} \geq\left\{\begin{array}{lll}
L-M & \text { for } & n=2,3, \cdots t \\
d_{t+1} & \text { for } & n=t+1, \cdots
\end{array}\right.
$$

The above results are best possible for the given function

$$
f(z)=z+\frac{L-M}{d_{t+1}} z^{t+1},
$$

where $d_{t+1}$ is given by (22).

Proof. Let us denote

$$
\begin{aligned}
\Phi_{1}(z) & =\frac{d_{t+1}}{L-M}\left\{\frac{f(z)}{S_{t}(f)}-\left(1-\frac{L-M}{d_{t+1}}\right)\right\} \\
& =1+\frac{\frac{d_{t+1}}{L-M} \sum_{n=t+1}^{\infty} a_{n} z^{n}}{z+\sum_{n=2}^{t} a_{n} z^{n}+\sum_{n=2}^{\infty} b_{n} \overline{z^{n}}} .
\end{aligned}
$$

The inequality (19) will be obtained if we can prove $\operatorname{Re}\left\{\Phi_{1}(z)\right\}>0$ and for this we need to prove the below inequality:

$$
\left|\frac{\Phi_{1}(z)-1}{\Phi_{1}(z)+1}\right| \leq 1 .
$$

In other words

$$
\left|\frac{\Phi_{1}(z)-1}{\Phi_{1}(z)+1}\right| \leq \frac{\frac{d_{t+1}}{L-M} \sum_{n=t+1}^{\infty}\left|a_{n}\right|}{2-2\left(\sum_{n=2}^{t}\left|a_{n}\right|+\sum_{n=2}^{\infty}\left|b_{n}\right|\right)-\frac{d_{t+1}}{L-M} \sum_{n=t+1}^{\infty}\left|a_{n}\right|} \leq 1,
$$


if and only if

$$
\sum_{n=2}^{t}\left|a_{n}\right|+\sum_{n=2}^{\infty}\left|b_{n}\right|+\frac{d_{t+1}}{L-M} \sum_{n=t+1}^{\infty}\left|a_{n}\right| \leq 1
$$

By the virtue of (8), it would be enough to ensure that the left hand side of (24) is bounded above by

$$
\sum_{n=2}^{\infty} \frac{d_{n}}{L-M}\left|a_{n}\right|+\sum_{n=2}^{\infty} \frac{d_{n}}{L-M}\left|b_{n}\right|
$$

and this is equal to

$$
\begin{gathered}
\sum_{n=2}^{t} \frac{d_{n}-L+M}{(L-M)}\left|a_{n}\right|+\sum_{n=2}^{\infty} \frac{d_{n}-L+M}{L-M}\left|b_{n}\right| \\
+\sum_{n=t+1}^{\infty} \frac{d_{n}-d_{t+1}}{L-M}\left|a_{n}\right| \geq 0
\end{gathered}
$$

which is true due to relation (22). To prove that the function

$$
f(z)=z+\frac{L-M}{d_{t+1}} z^{t}
$$

provides the best possible result. We note for $z=r e^{i \frac{\pi}{t}}$ that

$$
\begin{aligned}
\frac{f(z)}{S_{t}(f)} & =1+\frac{L-M}{d_{t+1}} z^{t} \rightarrow 1-\frac{L-M}{d_{t+1}} r^{t} \\
& =\frac{d_{t+1}-L+M}{d_{t+1}} .
\end{aligned}
$$

Now to prove (20), let us write

$$
\begin{aligned}
\Phi_{2}(z) & =\frac{d_{t+1}+L-M}{L-M}\left\{\frac{S_{t}(f)}{f(z)}-\left(1-\frac{L-M}{d_{t+1}+L-M}\right)\right\} \\
& =1-\frac{\frac{d_{t+1}+(L-M)}{(L-M)} \sum_{n=t+1}^{\infty} a_{n} z^{n}}{z+\sum_{n=2}^{\infty} a_{n} z^{n}+\sum_{n=2}^{\infty} b_{n} \overline{z^{n}}} .
\end{aligned}
$$

Then

$$
\left|\frac{\Phi_{2}(z)-1}{\Phi_{2}(z)+1}\right| \leq \frac{\frac{d_{t+1}+L-M}{L-M} \sum_{n=t+1}^{\infty}\left|a_{n}\right|}{2-2\left(\sum_{n=2}^{t}\left|a_{n}\right|+\sum_{n=2}^{\infty}\left|b_{n}\right|\right)-\frac{d_{t+1}-L+M}{L-M} \sum_{n=t+1}^{\infty}\left|a_{n}\right|} \leq 1,
$$

if and only if

$$
\sum_{n=2}^{t}\left|a_{n}\right|+\sum_{n=2}^{\infty}\left|b_{n}\right|+\frac{d_{t+1}}{L-M} \sum_{n=t+1}^{\infty}\left|a_{n}\right| \leq 1
$$

The inequality (25) is true only if the left side of this inequality is bounded above by

$$
\sum_{n=2}^{\infty} \frac{d_{n}}{L-M}\left|a_{n}\right|+\sum_{n=2}^{\infty} \frac{d_{n}}{L-M}\left|b_{n}\right|,
$$

and hence the proof is completed due to the use of (8).

Theorem 4. Let $f=\mathfrak{h}+\overline{\mathfrak{g}}$, where $\mathfrak{h}$ and $\mathfrak{g}$ are given by (2). If $f$ satisfies the condition (8), then 


$$
\operatorname{Re}\left(\frac{f(z)}{S_{l}(f)}\right) \geq \frac{d_{l+1}-L+M}{d_{l+1}}
$$

and

$$
\operatorname{Re}\left(\frac{S_{l}(f)}{f(z)}\right) \geq \frac{d_{l+1}}{d_{l+1}+L-M^{\prime}}
$$

where $d_{n}$ is given by (21) and

$$
d_{n} \geq\left\{\begin{array}{lll}
L-M & \text { for } & n=2,3, \cdots l \\
d_{l+1} & \text { for } & n=l+1, \cdots
\end{array}\right.
$$

Equalities are obtain by using the function

$$
f(z)=z+\frac{L-M}{d_{l+1}} \overline{z^{l+1}} .
$$

Proof. The evidence of this specific result is analogous to that of Theorem 3, and is therefore exempted.

Theorem 5. Let $f=\mathfrak{h}+\overline{\mathfrak{g}}$ be of the form (1). If $f$ fulfills the inequality (8), then

$$
\operatorname{Re}\left(\frac{f(z)}{S_{t, l}(f)}\right) \geq \frac{d_{t+1}-(L-M)}{d_{t+1}}
$$

and

$$
\operatorname{Re}\left(\frac{S_{t, l}(f)}{f(z)}\right) \geq \frac{d_{t+1}}{d_{t+1}+(L-M)}
$$

where $d_{n}$ is given by (21). Equalities are obtained for the function given in (23).

Proof. In order to show (30), let us write

$$
\begin{aligned}
\Phi_{3}(z) & =\frac{d_{t+1}}{(L-M)}\left\{\frac{f(z)}{S_{t, l}(f)}-\left(1-\frac{(L-M)}{d_{t+1}}\right)\right\} \\
& =1+\frac{\frac{d_{t+1}}{(L-M)}\left(\sum_{n=t+1}^{\infty} a_{n} z^{n}+\sum_{n=l+1}^{\infty} b_{n} \overline{z^{n}}\right)}{z+\sum_{n=2}^{t} a_{n} z^{n}+\sum_{n=2}^{l} b_{n} \overline{z^{n}}} .
\end{aligned}
$$

Thus for the proof of inequality (19) it is enough to prove the following inequality:

$$
\left|\frac{\Phi_{3}(z)-1}{\Phi_{3}(z)+1}\right| \leq 1 .
$$

Now consider the left hand side of (32) and then by simple computation, we have

$$
\left|\frac{\Phi_{3}(z)-1}{\Phi_{3}(z)+1}\right| \leq \frac{\frac{d_{t+1}}{(L-M)}\left(\sum_{n=t+1}^{\infty} a_{n} z^{n}+\sum_{n=l+1}^{\infty} b_{n} \overline{z^{n}}\right)}{2-2\left(\sum_{n=2}^{t}\left|a_{n}\right|+\sum_{n=2}^{l}\left|b_{n}\right|\right)-\frac{d_{t+1}}{L-M}\left(\sum_{n=t+1}^{\infty}\left|a_{n}\right|+\sum_{n=l+1}^{\infty}\left|b_{n}\right|\right)} .
$$

Since, from the use of (8), we observe that the denominator of the last inequality is positive. Thus the right hand side of the last inequality is bounded above by one if and only if the following inequality hold 


$$
\sum_{n=2}^{t}\left|a_{n}\right|+\sum_{n=2}^{l}\left|b_{n}\right|+\frac{d_{t+1}}{L-M}\left(\sum_{n=t+1}^{\infty}\left|a_{n}\right|+\sum_{n=l+1}^{\infty}\left|b_{n}\right|\right) \leq 1 .
$$

Finally, to prove the inequality in (30), it suffices to show that the left hand side of (33) is bounded by

$$
\sum_{n=2}^{\infty} \frac{d_{n}}{L-M}\left|a_{n}\right|+\sum_{n=2}^{\infty} \frac{d_{n}}{L-M}\left|b_{n}\right|,
$$

which is equivalent to

$$
\sum_{n=2}^{t} \frac{d_{n}-(L-M)}{(L-M)}\left|a_{n}\right|+\sum_{n=2}^{l} \frac{d_{n}-(L-M)}{(L-M)}\left|b_{n}\right|+\frac{d_{n}-d_{t+1}}{L-M}\left(\sum_{n=t+1}^{\infty}\left|a_{n}\right|+\sum_{n=l+1}^{\infty}\left|b_{n}\right|\right) \geq 0,
$$

and the last inequality is true because of (28). For the sharpness, let's consider

$$
f(z)=z+\frac{L-M}{d_{t+1}} z^{t},
$$

which provides the sharp result. We note for $z=r e^{i \frac{\pi}{t}}$ that

$$
\frac{f(z)}{S_{t}(f)}=1+\frac{L-M}{d_{t+1}} z^{t} \rightarrow 1-\frac{L-M}{d_{t+1}} r^{t}(r \rightarrow 1) .
$$

Similarly we obtain the assertion (31).

Theorem 6. Let $f=\mathfrak{h}+\overline{\mathfrak{g}}$ with $\mathfrak{h}$ and $\mathfrak{g}$ are expressed by (2). If $f$ satisfies the condition (8), then

$$
\operatorname{Re}\left(\frac{f(z)}{S_{t, l}(f)}\right) \geq \frac{d_{l+1}-(L-M)}{d_{l+1}}
$$

and

$$
\operatorname{Re}\left(\frac{S_{l}(f)}{f(z)}\right) \geq \frac{d_{l+1}}{d_{l+1}+(L-M)},
$$

where $d_{n}$ is given by (28). These estimates are sharp for the function given by (29).

Proof. The proof is identical to that of Theorem 5 proof and is thus excluded.

4. Further Properties of a class $\mathcal{S}_{\tau}^{*}(x, y, q, L, M)$

Theorem 7. If $f \in \mathcal{S}_{\mathcal{\tau}}^{*}(x, y, q, L, M)$, then for $|z|=r$

$$
|f(z)| \leq r+\frac{L-M}{\sigma_{2}} r^{2},
$$

and

$$
|f(z)| \geq r-\frac{L-M}{\sigma_{2}} r^{2} .
$$

Proof. Let $f=\mathfrak{h}+\overline{\mathfrak{g}} \in \mathcal{S}_{\tau}^{*}(x, y, q, L, M)$ with $\mathfrak{h}$ and $\mathfrak{g}$ be the form (2). Using Theorem 2 and letting

$$
\begin{aligned}
|f(z)| & \leq|z|+\sum_{n=2}^{\infty}\left(\left|a_{n}\right|+\left|b_{n}\right|\right)\left|z^{n}\right| . \\
& \leq|z|+\frac{1}{\sigma_{2}}|z|^{2} \sum_{n=2}^{\infty}\left(\sigma_{n}\left|a_{n}\right|+v_{n}\left|b_{n}\right|\right) \\
& \leq|z|+\frac{L-M}{\sigma_{2}}|z|^{2} .
\end{aligned}
$$


This proves (36). On similar lines one can easily achieve (37).

Theorem 8. A function $f \in \mathcal{S}_{\tau}^{*}(x, y, q, L, M)$ if and only if

$$
f(z)=\sum_{n=2}^{\infty} x_{n} \mathfrak{h}_{n}(z)+y_{n} \mathfrak{g}_{n}(z)
$$

where

$$
\begin{aligned}
\mathfrak{h}(z) & =z, \\
\mathfrak{h}_{n}(z) & =z-\frac{L-M}{\sigma_{n}} z^{n} \text { for } n \in \mathbb{N} \backslash\{1\}, \\
\mathfrak{g}_{n}(z) & =z-\frac{L-M}{v_{n}} \overline{z^{n}} \text { for } n \in \mathbb{N} \backslash\{1\},
\end{aligned}
$$

and $x_{n}, y_{n} \geq 0$, for $n \geq 2$ such that

$$
\sum_{n=2}^{\infty}\left(x_{n}+y_{n}\right)=1
$$

In particular the points $\left\{\mathfrak{h}_{n}\right\},\left\{\mathfrak{g}_{n}\right\}$ are called the extreme points of the closed convex hull of the class $\mathcal{S}_{\tau}^{*}(x, y, q, L, M)$ denoted by $\operatorname{clcoS}_{\tau}^{*}(x, y, q, L, M)$.

Proof. Let $f$ be given by (38). Then, using (39), we can easily attain

$$
f(z)=z-\sum_{n=2}^{\infty}\left(\frac{L-M}{\sigma_{n}} x_{n} z^{n}+\frac{L-M}{v_{n}} y_{n} \bar{z}^{n}\right),
$$

which by Theorem 2 proves that $f \in \mathcal{S}_{\tau}^{*}(x, y, q, L, M)$. Since for this function

$$
\sum_{n=2}^{\infty}\left(\frac{\sigma_{n}}{L-M} \frac{L-M}{\sigma_{n}} x_{n}+\frac{v_{n}}{L-M} \frac{L-M}{v_{n}} y_{n}\right)=\sum_{n=2}^{\infty}\left(x_{n}+y_{n}\right) \leq 1
$$

Thus $f \in \operatorname{clcoS}_{\tau}^{*}(x, y, q, L, M)$. Conversely, let $f=\mathfrak{h}+\overline{\mathfrak{g}} \in \mathcal{S}_{\tau}^{*}(x, y, q, L, M)$, where $\mathfrak{h}$ and $\mathfrak{g}$ are of the form (14). Set

$$
x_{n}=\frac{\sigma_{n}}{L-M}\left|a_{n}\right|, y_{n}=\frac{v_{n}}{L-M}\left|b_{n}\right| .
$$

Then by using (39) along with the given hypothesis, we have

$$
\begin{aligned}
f(z) & =z+\sum_{n=2}^{\infty} a_{n} z^{n}+\sum_{n=2}^{\infty} \overline{b_{n} z^{n}} \\
& =z-\sum_{n=2}^{\infty}\left|a_{n}\right| z^{n}-\sum_{n=2}^{\infty}\left|b_{n}\right| \overline{z^{n}} \\
& =z-\sum_{n=2}^{\infty} x_{n} \frac{L-M}{\sigma_{n}} z^{n}-\sum_{n=2}^{\infty} y_{n} \frac{L-M}{v_{n}} \bar{z}^{n} \\
& =z-\sum_{n=2}^{\infty} x_{n}\left\{z-\mathfrak{h}_{n}(z)\right\}-\sum_{n=2}^{\infty} y_{n}\left\{z-\mathfrak{g}_{n}(z)\right\} \\
& =\left(1-\sum_{n=2}^{\infty} x_{n}+y_{n}\right) z+\sum_{n=2}^{\infty}\left\{x_{n} \mathfrak{h}_{n}(z)+y_{n} \mathfrak{g}_{n}(z)\right\} \\
& =\sum_{n=2}^{\infty}\left\{x_{n} \mathfrak{h}_{n}(z)+y_{n} \mathfrak{g}_{n}(z)\right\}
\end{aligned}
$$

which is of the form (38). This proves Theorem 8 . 
Theorem 9. If $f_{1}, f_{2} \in \mathcal{S}_{\tau}^{*}(x, y, q, L, M)$, then $f_{1} * f_{2} \in \mathcal{S}_{\tau}^{*}(x, y, q, L, M)$.

Proof. Let

$$
f_{1}(z)=z-\sum_{n=2}^{\infty}\left|a_{n}\right| z^{n}-\sum_{n=2}^{\infty}\left|b_{n}\right| \bar{z}^{n}
$$

and

$$
f_{2}(z)=z-\sum_{n=2}^{\infty}\left|A_{n}\right| z^{n}-\sum_{n=2}^{\infty}\left|B_{n}\right| \bar{z}^{n}
$$

Then

$$
\left(f_{1} * f_{2}\right)(z)=z+\sum_{n=2}^{\infty}\left|A_{n}\right|\left|a_{n}\right| z^{n}+\sum_{n=2}^{\infty}\left|B_{n}\right|\left|b_{n}\right| \bar{z}^{n} .
$$

Since $f_{2} \in \mathcal{S}_{\tau}^{*}(x, y, q, L, M)$, by Theorem 2 , we have $\left|A_{n}\right| \leq 1$ and $\left|B_{n}\right| \leq 1$. Therefore

$$
\frac{1}{L-M} \sum_{n=2}^{\infty} \sigma_{n}\left|A_{n}\right|\left|a_{n}\right|+v_{n}\left|B_{n}\right|\left|b_{n}\right| \leq \frac{1}{L-M} \sum_{n=2}^{\infty} \sigma_{n}\left|a_{n}\right|+v_{n}\left|b_{n}\right| \leq 1 .
$$

By Theorem 2, it follows that $f_{1} * f_{2} \in \mathcal{S}_{\tau}^{*}(x, y, q, L, M)$.

Theorem 10. The class $\mathcal{S}_{\tau}^{*}(x, y, q, L, M)$ is closed under convex combination.

Proof. For $j \in \mathbb{N}$, let $f_{j} \in \mathcal{S}_{\tau}^{*}(x, y, q, L, M)$ be given by

$$
f_{j}(z)=z-\sum_{n=2}^{\infty}\left|a_{n, j}\right| z^{n}-\sum_{n=2}^{\infty}\left|b_{n, j}\right| \bar{z}^{n} .
$$

Then by (8), we have

$$
\sum_{n=2}^{\infty}\left\{\frac{\sigma_{n}\left|a_{n, j}\right|+v_{n}\left|b_{n, j}\right|}{L-M}\right\} \leq 1
$$

For $\sum_{j=1}^{\infty} \xi_{j}=1,0 \leq \xi_{j} \leq 1$, the convex combination of $f_{j}$ is

$$
\sum_{j=1}^{\infty} \xi_{j} f_{j}(z)=z-\sum_{n=2}^{\infty}\left(\sum_{j=1}^{\infty} \xi_{j}\left|a_{n, j}\right|\right) z^{n}-\sum_{n=2}^{\infty}\left(\sum_{j=1}^{\infty} \xi_{j}\left|b_{n, j}\right|\right) \bar{z}^{n} .
$$

Then on using Theorem 2, we can write

$$
\begin{aligned}
\sum_{n=2}^{\infty}\left(\sum_{j=1}^{\infty} \sigma_{n} \xi_{j}\left|a_{n, j}\right|+\sum_{i=1}^{\infty} v_{n} \xi_{j}\left|b_{n, j}\right|\right) & \leq \sum_{j=1}^{\infty} \xi_{j}\left\{\sum_{n=2}^{\infty}\left(\sigma_{n}\left|a_{n, j}\right|+v_{n}\left|b_{n, j}\right|\right)\right\} \\
& \leq(L-M) \sum_{j=1}^{\infty} \xi_{j}=(L-M),
\end{aligned}
$$

and so $\sum_{j=1}^{\infty} \xi_{j} f_{j}(z) \in \mathcal{S}_{\tau}^{*}(x, y, q, L, M)$.

\section{Conclusions}

Using the concepts of quantum calculus, we introduced some new subfamilies of Janowski harmonic mapping with symmetrical points. We studied some useful problems, including necessary and sufficient conditions, distortion limits, problems with partial sums, convolutions and convexity conditions for the newly defined classes of functions. For these classes, problems like Topological properties, integral mean inequalities, and their applications are open for the researchers to determine. Further, these problems can be studied for classes of meromorphic type harmonic functions as well. 
Author Contributions: Conceptualization, M.A. and H.M.S.; Formal analysis, M.A. and S.A.; Funding acquisition, O.B.; Investigation, M.A.; Methodology, S.A.K. and S.A.; Software, S.A.K.; Supervision, M.A., H.M.S. and O.B.; Visualization, S.A.; Writing-original draft, M.A. and S.A.K.; Writing-review \& editing, M.A. and H.M.S. All authors have read and agreed to the published version of the manuscript.

Funding: This project was funded by the Deanship of Scientific Research (DSR), King Abdulaziz University, under grant No. (DF-764-830-1441). The authors, therefore, gratefully acknowledge DSR technical and financial support.

Conflicts of Interest: The authors declare no conflict of interest.

\section{References}

1. Clunie, J.; Small, T.S. Harmonic univalent functions. Ann. Acad. Sci. Fen. Ser. A I Math. 1984, 9, 3-25. [CrossRef]

2. Small, T.S. Constants for planar harmonic mappings. J. Lond. Math. Soc. 1990, 2, 237-248. [CrossRef]

3. Dziok, J. On Janowski harmonic functions. J. Appl. Anal. 2015, 21, 99-107. [CrossRef]

4. Janowski, W. Some extremal problems for certain families of analytic functions I. Ann. Polon. Math. 1973, 28, 297-326. [CrossRef]

5. Jahangiri, J.M. Harmonic functions starlike in the unit disk. J. Math. Anal. Appl. 1999, 235, 470-477. [CrossRef]

6. Ahuja, O.P. Planar harmonic univalent and related mappings. J. Inequal. Pure Appl. Math. 2005, 6, 1-18.

7. Jahangiri, J.M.; Kim, Y.C.; Srivastava, H.M. Construction of a certain class of harmonic close-to-convex functions associated with the Alexander integral transform. Integral Trans. Spec. Funct. 2003, 14, $237-242$. [CrossRef]

8. Dziok, J.; Darus, M.; Sokół, J.; Bulboacă, T. Generalizations of starlike harmonic functions. C. R. Math. 2016, 354, 13-18. [CrossRef]

9. Lewy, H. On the non-vanshing of the Jacobian in certain one-to-one mappings. Bull. Am. Math. Soc. 1936, 42, 689-692. [CrossRef]

10. Jahangiri, J.M. Coefficient bounds and univalence criteria for harmonic functions with negative coefficients. Ann. Univ. Marie Curie-Sklodowska Sect. A 1998, 52, 57-66.

11. Jackson, F.H. On $q$-functions and a certain difference operator. Trans. R. Soc. Edinburgh. 1909, 46, $253-281$. [CrossRef]

12. Jackson, F.H. On q-definite integrals. Q. J. Pure Appl. Math. 1910, 41, 193-203.

13. Aral, A.; Gupta, V.; Agarwal, R.P. Applications of q-Calculus in Operator Theory; Springer: New York, NY, USA, 2013.

14. Ernst, T. A Comprehensive Treatment of q-Calculus; Springer: Basel, Switzerland; Heidelberg, Germany; New York, NY, USA; Dordrecht, Netherlands; London, UK, 2012.

15. Ismail, M.E.H.; Merkes, E.; Styer, D. A generalization of starlike functions. Complex Var. Theory Appl. 1990, 14, 77-84. [CrossRef]

16. Agrawal, S.; Sahoo, S.K. A generalization of starlike functions of order alpha. Hokkaido Math. J. 2017, 46, 15-27. [CrossRef]

17. Srivastava, H.M.; Tahir, M.; Khan, B.; Ahmad, Q.Z.; Khan, N. Some general families of $q$-starlike functions associated with the Janowski functions. Filomat 2019, 33, 2613-2626. [CrossRef]

18. Ahmad, K.; Arif, M.; Liu, J.-L. Convolution properties for a family of analytic functions involving $q$-analogue of Ruscheweyh differential operator. Turk. J. Math. 2019, 43, 1712-1720. [CrossRef]

19. Arif, M.; Srivastava, H.M.; Umar, S. Some applications of a $q$-analogue of the Ruscheweyh type operator for multivalent functions. Rev. Real Acad. Cienc. Exact. Físic. Nat. Ser. A Matemát. 2019, 113, 1211-1221. [CrossRef]

20. Shi, L.; Khan, Q.; Srivastava, G.; Liu, J.-L.; Arif, M. A study of multivalent $q$-starlike functions connected with circular domain. Mathematics 2019, 7, 670. [CrossRef]

21. Shi, L.; Raza, M.; Javed, K.; Hussain, S.; Arif, M. Class of analytic functions defined by $q$-integral operator in a symmetric region. Symmetry 2019, 11, 1042. [CrossRef]

22. Srivastava, H.M.; Khan, B.; Khan, N.; Ahmad, Q.Z. Coefficient inequalities for $q$-starlike functions associated with the Janowski functions. Hokkaido Math.J. 2019, 48, 407-425. [CrossRef]

23. Srivastava, H.M.; Tahir, M.; Khan, B.; Ahmad, Q.Z.; Khan, N. Some general classes of $q$-starlike functions associated with the Janowski functions. Symmetry 2019, 11, 292. [CrossRef]

24. Srivastava, H.M. Univalent functions, fractional calculus, and associated generalized hypergeometric functions. In Univalent Functions, Fractional Calculus, and Their Applications; Srivastava, H.M., Owa, S., Eds.; Halsted Press: Chichester, UK, 1989; pp. 329-354. 
25. Ul-Haq, M.; Raza, M.; Arif, M.; Khan, Q.; Tang, H. q-analogue of differential subordinations. Mathematics 2019, 7, 724. [CrossRef]

26. Sakaguchi, K. On a certain univalent mapping. J. Math. Soc. Jpn. 1959, 2, 72-75. [CrossRef]

27. Cho, N.E.; Dziok, J. Harmonic starlike functions with respect to symmetric points. Axioms 2020, 9, 3. [CrossRef]

28. Ahuja, O.P.; Jahangiri, J.M. Sakaguchi-type harmonic univalent functions. Sci. Math. Jpn. 2004, 59, $163-168$.

29. Al Amoush, A.G.; Darus, M. Harmonic starlike functions with respect to symmetric points. Matematika 2016, 32, 121-131.

30. Al-Khal, R.A.; Al-Kharsani, H.A. Salagean-type harmonic univalent functions with respect to symmetric points. Aust. J. Math. Anal. Appl. 2007, 4, 1-13.

31. Janteng, A.; Halim, S.A. Properties of harmonic functions which are starlike of complex order with respect to symmetric points. Int. J. Contemp. Math. Sci. 2009, 4, 275-281.

32. Silverman, H. Harmonic univalent functions with negative coefficients. J. Math. Anal. Appl. 1998, 220, 283-289. [CrossRef]

(C) 2020 by the authors. Licensee MDPI, Basel, Switzerland. This article is an open access article distributed under the terms and conditions of the Creative Commons Attribution (CC BY) license (http:/ / creativecommons.org/licenses/by/4.0/). 